\title{
Lung Tumor Segmentation Using Electric Flow Lines for Graph Cuts
}

\author{
Hollensen, Christian; Cannon, George ; Cannon, Donald ; Bentzen, Søren ; Larsen, Rasmus
}

Published in:

Image Analysis and Recognition

Link to article, DOI:

10.1007/978-3-642-31298-4_25

Publication date:

2012

Link back to DTU Orbit

Citation $(A P A)$ :

Hollensen, C., Cannon, G., Cannon, D., Bentzen, S., \& Larsen, R. (2012). Lung Tumor Segmentation Using Electric Flow Lines for Graph Cuts. In Image Analysis and Recognition: 9th International Conference, ICIAR 2012 Aveiro, Portugal, June 25-27, 2012 Proceedings, Part II (pp. 206-213). Springer. Lecture Notes in Computer Science No. 7325 https://doi.org/10.1007/978-3-642-31298-4_25

\section{General rights}

Copyright and moral rights for the publications made accessible in the public portal are retained by the authors and/or other copyright owners and it is a condition of accessing publications that users recognise and abide by the legal requirements associated with these rights.

- Users may download and print one copy of any publication from the public portal for the purpose of private study or research.

- You may not further distribute the material or use it for any profit-making activity or commercial gain

- You may freely distribute the URL identifying the publication in the public portal 


\title{
Lung Tumor Segmentation Using Electric Flow Lines for Graph Cuts
}

\author{
Christian Hollensen ${ }^{1}$, George Cannon ${ }^{2}$, Donald Cannon ${ }^{2}$, Søren Bentzen ${ }^{2}$, and \\ Rasmus Larsen ${ }^{1}$ \\ 1 Technical University of Denmark, \\ Department of Informatics and Mathematical Modelling, Denmark, \\ chrho@imm.dtu.dk \\ 2 University of Wisconsin, Department of Human Oncology, WI, USA
}

\begin{abstract}
Lung cancer is the most common cause of cancer-related death. A common treatment is radiotherapy where the lung tumors are irradiated with ionizing radiation. The treatment is typically fractionated, i.e. spread out over time, allowing healthy tissue to recover between treatments and allowing tumor cells to be hit in their most sensitive phase. Changes in tumors over the course of treatment allows for an adaptation of the radiotherapy plan based on 3D computer tomography imaging. This paper introduces a method for segmentation of lung tumors on consecutive computed tomography images. These images are normally only used for correction of movements. The method uses graphs based on electric flow lines. The method offers several advantages when trying to replicate manual segmentations. The method gave a dice coefficient of 0.85 and performed better than level set methods and deformable registration.
\end{abstract}

Keywords: Electric flow line, segmentation, lung tumor, graph cut

\section{Introduction}

Treatment response is important in lung cancer treatment. Without treatment response it is impossible to assess the progress or lack of progress of a treatment. With the continued increase of treatment strategies that is available today the possibility of earlier treatment response becomes more and more pertinent. If the patient does not respond adequately to a certain treatment early prediction can spare the patient unnecessary toxic treatment and be used to change treatment strategy. During the last 4 decades 3-dimensional imaging modalities has become one of the most important tools in the clinical assessment of cancer.

Positron emission tomography (PET) has shown to be correlated with treatment outcome [1]. But it is not a conventional tool for early treatment response. Helical Tomotherapy is an external beam radiation therapy system which has a megavoltage $\mathrm{x}$-ray source which makes it capable of making megavoltage computed tomography (MVCT) images. These images are used to provide image 
guided radiotherapy (IGRT) imaging the target volume to allow adjustment of the patient to optimize treatment. These images potentially includes information about treatment response [2], [3]. But the quantity (up to 30 fractions per patient) of these images makes manual contouring of gross tumor volume (GTV) unfeasible for clinics.

Several approaches has been developed to segment tumors throughout treatment. Kuhnigk et al [4] developed a method for segmentation of lung lesions and estimation of partial volume effect, Fetita et al. [5] developed a method for lung nodule segmentation and Faggiano et al. [6] developed a registration method to estimate anatomical modification during radiation treatment. Some of these methods are only applicable for kilovoltage computed tomography (KVCT) which are only available for the initial planning scan of these patients. Furthermore, these methods require human interaction for each image sequence.

In this paper we propose a graph based method [7] which uses the principle of electric flow line (EFL) theory [8], [9] to segment lung tumors on MVCT images. The method is novel in regard to utilizing available temporal images of the tumor and makes graphs with unique paths which are transferable from one fraction to another. Overlap and distance to manual contours are calculated and compared to other automatic methods for segmentation of 3-dimensional images.

\section{Methodology}

We assume an initial pre-treatment scan annotated by manually contouring each slice of the GTV. This will typically be a KVCT scan. At the following treatment sessions a series of scans are then acquired. These will typically be a MVCT scans. For each scan in this sequence we want to transfer the GTV outline from the previous scan rigidly to the current scan and to non-rigidly deform this outline to fit to the intensity patterns in the current image. In order to avoid self-intersections we will make this deformation along a graph following EFL derived from simulating an artificial charge at the outline [8], [9] and using a graph cut method to find an optimal tumor outline with respect to smoothness of the outline and correspondence to the underlying image patterns.

\subsection{Rigid Registration}

The rigid registration between scans is performed using correlation as a similarity measure for the volume within $2 \mathrm{~cm}$ of the GTV [10]. In the practical application of this method a manual contour was transferred from the KVCT scan to the first MVCT scan. On all following MVCT scans the automatic segmentation was transferred from one scan to the following using the same procedure.

\section{$2.2 \quad$ Electric Flow Lines}

The initial segmentation from the previous scan is used to generate the EFLs. The electric flow should stem from an electric potential on the surface of the segmentation. The potential, $E$, is defined as: 


$$
E(x)=\int Q(\tilde{x}) R(\tilde{x}-x) d \tilde{x},
$$

where $x$ is the position at which the flow is evaluated and $Q$ is a function which is 1 on the surface and 0 at all other positions. $R$ is the potential at the position $x$ coming from the potential at $\tilde{x}$ defined by Coulombs law as:

$$
R(r)=\frac{r}{4 \pi \epsilon \cdot\|r\|^{3}},
$$

where $r$ is the direction vector from one potential to the point evaluated, $\epsilon$ is the electric constant which along with the $4 \pi \epsilon$ constant is ignored in practical computations. The surface potential was discretized by performing a Delauney triangulation of the segmentation points and placing a charge in the barycenter of each triangle. The potential of each triangle was set to its part of the total surface area.

Hereafter an electric line was initiated at each triangle barycenter and iteratively computed in an inwards and outwards direction using the above equations. For the first iteration the potential at the position was neglected because of infinite influence on the potential at the position. Instead an initial step in the direction of the triangle plane normal was used. The image values were extracted along each of the electric flow lines on both the former tumor scan and the current scan on which a segmentation is sought. The correlation between the flow lines from the former and current scan is calculated as a measure of accordance for each position along the EFLs.

\subsection{Graph Construction}

A graph is constructed with vertices, $V_{i, j}$ at each of the positions along the EFLs. $j$ corresponds to the individual EFLs or columns and $i$ corresponds to each of the positions along it. The graph consists of two types of edges, intraand inter-column edges. The intra-column edges account for the likelihood that the surface is located at that location. It is formulated as

$$
\begin{aligned}
& E_{\text {intra }}(i \rightarrow i-1, j)=\frac{1}{1+e^{c(i)}} \\
& E_{\text {intra }}(i \rightarrow i+1, j)=\infty
\end{aligned}
$$

where $c(i)$ is the correlation value of the $i$ th position along the EFL. A graph consisting only of the intra-column edges would have an optimal solution giving the positions with the highest correlation values. The intercolumn edges are implemented to ensure a smooth segmentation where the segmentation of one EFL is consistent with the surrounding EFLs. The inter-column edges are formulated as

$$
E_{\text {inter }}(i, k, j, l)=g_{i, k}(j, l),
$$




\section{Intra-column}

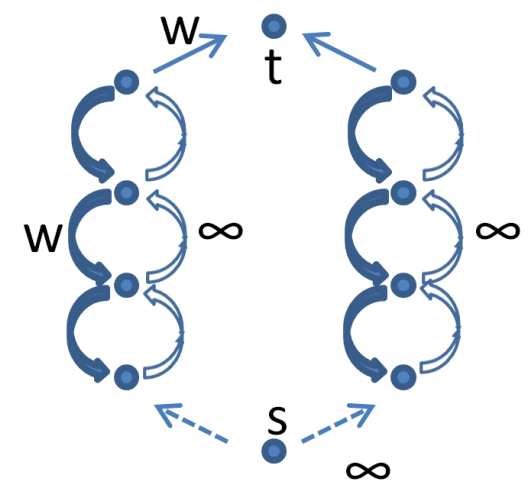

Inter-column

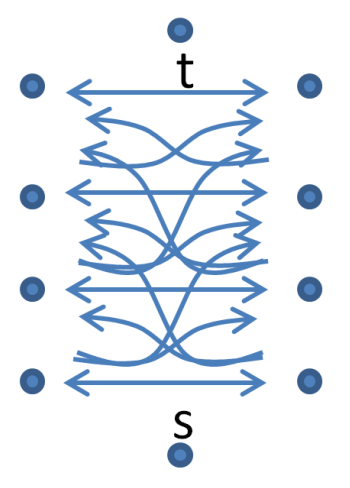

Fig. 1. Illustration of the edges in the graph. The $s$ and $t$ node are respectively the source and sink. In our implementation there are more layers in each column and there are edges with more than two layer difference.

$$
g_{i, k}(j, l)= \begin{cases}0 & \text { if } \operatorname{dist}(i, k)>1 \\ 0 & \text { if } i=k \\ 0 & \text { if } j<l \\ w \cdot e^{\frac{-(j-l)}{c}} & \text { else }\end{cases}
$$

where $i$ and $k$ are line indices referring to EFLs, $j$ and $l$ are position indices on the the EFL and $\operatorname{dist}(i, k)$ is the triangulation length between two vertices, which is equal to one when two triangles are interconnected. $g$ is the weighting parameter between EFLs, $w$ is the weight constant between EFL and $c$ is a normalization constant. The edges are illustrated in Fig. 1. The specific segmentation solution was found by solving the minimum cut problem on the graph [11].

\subsection{Graph Parameters}

All parameters of the graph were optimised on a spherical phantom with 2 hemispheres and 2 pyramids located on the surface. The phantom had a contour at $0.1 \mathrm{~mm}$ from the surface in the image to account for a manual contour not lying exact on the gradient border. The phantom was simulated with a radius variance of 0.2 , with different translations, rotations and signal-to-noise levels. A grid search was performed for different parameter settings. The parameters giving the highest dice coefficient were chosen for the actual tumor segmentations.

\subsection{Evaluating Results}

A segmentation for the MVCT scans was extracted using each of the two initial manual scans from the planning KVCT scan resulting in 6 new automatic 
segmentations for each patient. To evaluate the segmentation results the new segmentations were compared with both of the manual segmentations on the MVCT scans using dice coefficient, Hausdorff distance and mean distance. The images were also segmented using a level set method [12] and deformable registration [10] for comparison. A paired t-test was performed to assess significant difference from the novel method on a basis of 0.05 .

\section{Results}

\subsection{Experiment Data}

The study included images from ten non-small cell lung cancer (NSCLC) patients, 3 female and 7 males, chosen from a larger patient group from a dose escalation study [13]. The patients were treated between 2004 and 2009 at University of Wisconsin Hospital. They were all treated with radiotherapy delivered via helical tomotherapy. All patients were non-metastatic at stage IIIa, IIIb or recurrent histologically confirmed NSCLC with no prior thoracic radiation therapy. The patient selection criteria were a cranio-caudal length of less than $5 \mathrm{~cm}$ for the primary tumor the planning CT.

Primary planning KVCT scan images for all patients was exported along with the images of three MVCT scans from the Tomotherapy system from first, mid and last fraction (1, 13 and 25). The time span between planning KVCT and last fraction was between 34 and 41 days. The images were imported into Pinnacle (C) treatment planning system. If the MVCT image did not include the primary tumor, images from bordering fractions were chosen instead.

All MVCT images were manually registered to the KVCT image using Pinnacle (C) to replicate the usual clinical procedure. Primary GTV was contoured independently by two experienced radiation oncologists on on all patient images, 10 patients times 4 images. Provided along with the images were contours of normal tissue from the original treatment planning on the KVCT images. This means that in all 40 images were provided with 2 contours on each image. The contours and images are exported from Pinnacle (c) as Dicoms and imported to Matlab@ using CERR [14] where further processing was performed.

\subsection{Experiment Results}

It was possible to segment the tumor for all the patients on all of their images. Result examples for the novel segmentation can be seen in Fig. 2. The results are seen in Table 1. The novel method had significant better dice coefficient and mean difference than the two other methods using a paired t-test of 0.05 but not for Hausdorff distance. Looking at the volume as a treatment outcome the manual segmentations had a mean variation of $7.1 \%$ on the KVCT images and $8.5 \%$ on the MVCT images. The novel method had a mean variation of $4.5 \%$ for the volume on the MVCT images. 

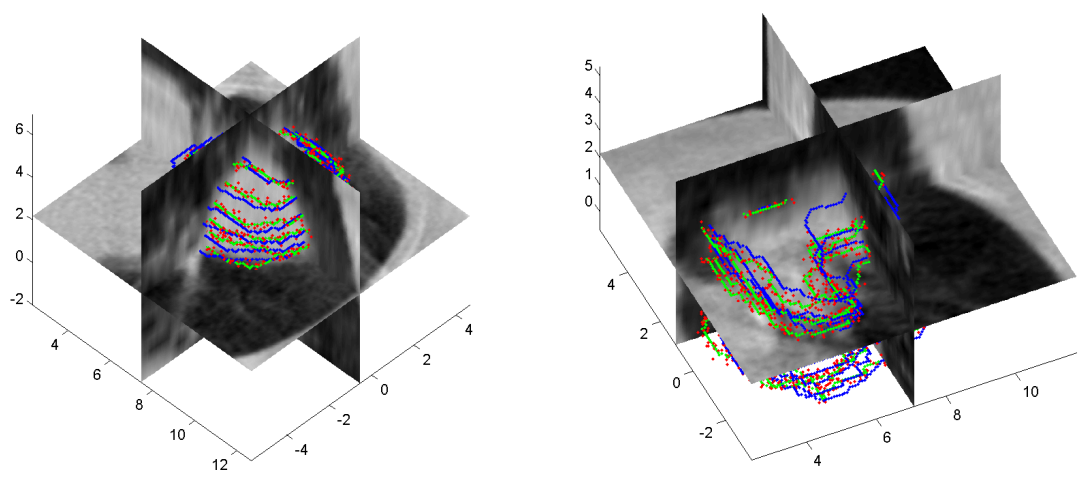

Fig. 2. Examples of the segmentation on a MVCT image. Green and blue points are the two different manual segmentations. The red point are the automatic segmentation of the novel method.

Table 1. The results of the different methods of segmentation. Seg. type: Segmentation type. Haus. Dis.: Hausdorff distance. Mean Dis.: Hausdorff distance. EFL: Electric flow line segmentation. Deformable: Deformable registration. * The manual segmentations are only compared pairwise to each other whereas the automatic segmentation are compared to both of the manual segmentations.

\begin{tabular}{|c|c|c|c|}
\hline Seg. Type & Dice & Haus. Dis. & Mean Dis. \\
\hline Manual $\star$ & $0.78 \pm 0.13$ & $0.8 \pm 0.7$ & $0.24 \pm 0.10$ \\
\hline EFL & $0.85 \pm 0.11$ & $0.7 \pm 0.5$ & $0.15 \pm 0.05$ \\
\hline Level set & $0.67 \pm 0.22$ & $0.9 \pm 0.6$ & $0.29 \pm 0.09$ \\
\hline Deformable & $0.73 \pm 0.20$ & $0.8 \pm 0.7$ & $0.22 \pm 0.08$ \\
\hline
\end{tabular}

\section{Discussion}

The method of parameter optimization should be mentioned as a first point of scrutiny. It is solely performed on a simulated phantom. The phantom is constructed ideally with normally distributed noise, rotations and translation of the whole phantom. The tumor image sequences have none of these characteristics. But even so it represented an acceptable method for finding segmentation parameters for the tumor segmentation. It would also be possible to find parameters by using manual segmentations on tumor images. But this would just find parameters to replicate one manual contourer. In this approach we created the phantom to have qualities which would suit a manual contourer generally.

It can be seen from table 1 that the novel method performs better compared to the other automatic methods here. The novel method performs better than the level set method and deformable registration. The level set method only includes image values and their gradients in its cost function. This can be a problem when attempting to repeat manual segmentations automatically because the manual segmentations do not necessarily follow image gradients. The same thing also 
applies for deformable registration. Even though the cost function was based on normalized correlation it does still have larger problems with circumference of its segmentation.

Segmentations of lung tumors have smaller variations than other tumor sites [15] it is still a hard tumor site because of movement of lungs and heart. These problems have been reduced by $4 \mathrm{D}$-visualization and breath hold techniques but it is still a significant problem for automatic segmentation methods. The quality of MVCTs does also increase the variation for manual contours as it can be seen on the difference in volume variation between KVCT and MVCT. One could apply better imaging techniques for these interfractional images to acquire a better outcome for the automatic segmentation methods. But this is not feasible in the current clinical setup. These images are taken to adjust beam and patient position but are not analyzed. This is both due to the amount of images and the lack of resources in the clinic.

The automatic contours are compared to manual segmentations in lack of better segmentations. An optimal comparison would be to the actual tumor

volumes. This is feasible in some tumor sites but it is not feasible in treatments with several fraction.

The novel method does have some shortcomings. It does expect some continuation of topology which for our images is optimal, because these tumors tend to retain most of their topology throughout treatment. If the tumors were dividing into several volume components it would pose as a suboptimal solution.

\section{Conclusion}

A novel method for 3-dimensional segmentation of consecutive images have been implemented and have been shown to perform better than level set methods and deformable registration concerning overlap. The method is applicable on clinical images without any modification of clinical practice.

\section{References}

1. Weber, W. et al. Positron emission tomography in non-small-cell lung cancer: prediction of response to chemotherapy by quantitative assessment of glucose use. Journal of Clinical Oncology 21, 2651-2657 (July 2003).

2. Siker, M. L., Tomé, W. A. \& Mehta, M. P. Tumor volume changes on serial imaging with megavoltage CT for non-small-cell lung cancer during intensity-modulated radiotherapy: how reliable, consistent, and meaningful is the effect? International Journal of Radiation Oncology Biology Physics 66, 135-141 (Sept. 2006).

3. Bral, S. et al. Daily megavoltage computed tomography in lung cancer radiotherapy: correlation between volumetric changes and local outcome. International Journal of Radiation Oncology Biology Physics 80, 13381342 (Aug. 2011). 
4. Kuhnigk, J. M. et al. Morphological segmentation and partial volume analysis for volumetry of solid pulmonary lesions in thoracic CT scans. IEEE Trans. Medical Imaging 25, 417-434 (2006).

5. Fetita, C. I., F. Prêteux, F., Beigelman-Aubry, C. \& Grenier, P. 3D automated lung nodule segmentation in HRCT. LNCS 2879, 626-634 (2003).

6. Faggiano, E. et al. Validation of an elastic registration technique to estimate anatomical lung modification in non-small-cell lung cancer tomotherapy. Radiation Oncology 6, 31 (2011).

7. Wu, X. \& Chen, D. Optimal Net Surface Problems with Applications. LNCS. LNCS 2380, 775-775 (2002).

8. Yin, Y., Song, Q. \& Sonka, M. in Proceedings of the 7th IAPR-TC-15 International Workshop on Graph-Based Representations in Pattern Recognition (Venice, Italy, 2009), 334-342.

9. Petersen, J. et al. Optimal graph based segmentation using flow lines with application to airway wall segmentation. eng. Information Processing in Medical Imaging 22, 49-60 (2011).

10. Vester-Christensen, M., Erbou, S. G., Darkner, S. \& Larsen, R. Accelerated 3D image registration. SPIE Medical Imaging (2007).

11. Boykov, Y., Veksler, O. \& Zabih, R. Markov random fields with efficient approximations. IEEE Computer Society Conference on Computer Vision Pattern Recognition Proceedings, 648-655 (1998).

12. Jørgensen, P., Hansen, K., Larsen, R. \& Bowen, J. A framework for automatic segmentation in three dimensions of microstructural tomography data. Ultramicroscopy 110, 216-228 (Feb. 2010).

13. Adkison, J. B. et al. Dose escalated, hypofractionated radiotherapy using helical tomotherapy for inoperable non-small cell lung cancer: preliminary results of a risk-stratified phase I dose escalation study. eng. Technology in Cancer Research Treatment 7, 441-447 (2008).

14. Deasy, J. O., Blanco, A. I. \& Clark, V. H. CERR: a computational environment for radiotherapy research. eng. Medical Physics 30, 979-985 (2003).

15. Steenbakkers, R. J. H. M. et al. Reduction of observer variation using matched CT-PET for lung cancer delineation: a three-dimensional analysis. eng. International Journal of Radiation Oncology Biology Physics 64, $435-448$ (2006). 